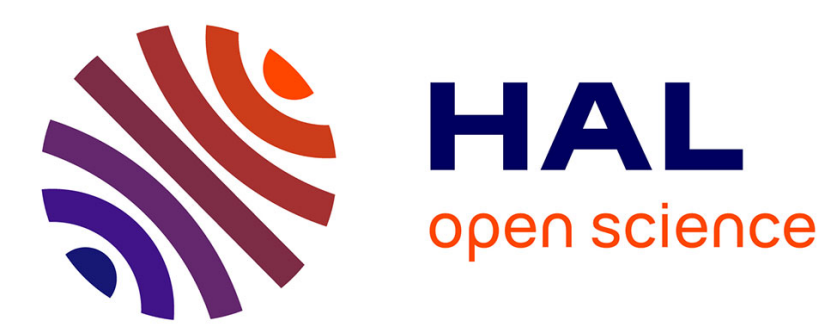

\title{
A Pair of Measures of Rotational Error for Axisymmetric Robot End-Effectors
}

\author{
Sébastien Briot, Ilian Bonev
}

\section{To cite this version:}

Sébastien Briot, Ilian Bonev. A Pair of Measures of Rotational Error for Axisymmetric Robot EndEffectors. Advances in Robot Kinematics, 11th International Symposium, Jun 2008, Batz-sur-Mer, France. hal-00451913

\section{HAL Id: hal-00451913 https://hal.science/hal-00451913}

Submitted on 25 Jun 2019

HAL is a multi-disciplinary open access archive for the deposit and dissemination of scientific research documents, whether they are published or not. The documents may come from teaching and research institutions in France or abroad, or from public or private research centers.
L'archive ouverte pluridisciplinaire HAL, est destinée au dépôt et à la diffusion de documents scientifiques de niveau recherche, publiés ou non, émanant des établissements d'enseignement et de recherche français ou étrangers, des laboratoires publics ou privés. 


\title{
A PAIR OF MEASURES OF ROTATIONAL ERROR FOR AXISYMMETRIC ROBOT END-EFFECTORS
}

\author{
Sébastien Briot, Ilian A. Bonev \\ Department of Automated Manufacturing Engineering \\ École de technologie supérieure (ÉTS), Montreal, Canada \\ sebastien.briot.1@ens.etsmtl.ca, ilian.bonev@etsmtl.ca
}

\begin{abstract}
This paper deals with the problem of representing the rotational error of spatial robots with three orientational degrees of freedom (DOF). Typically, the errors on each of three Euler angles defining the orientation of an endeffector are analysed separately. However, this is wrong since an accuracy measure should depend only on the "distance" between the nominal pose and the actual one, and not on the choice of reference frame in which these are represented. Several bi-invariant metrics for rotational error exist but are single-parameter and, by definition, disregard the shape of the robot endeffector. Yet, robot end-effectors are typically axisymmetric. Therefore, we propose a two-parameter measure of rotational errors that is better suited for such robot end-effectors.
\end{abstract}

Keywords: accuracy analysis, rotational errors, Euler angles, distance metrics.

\section{Introduction}

Knowing the exact positioning accuracy is an important challenge when designing a robot. Such an analysis is useful for optimization procedures occurring in the design stage of a new robot. Many tools have been developed in order to promptly estimate the accuracy of a robot. The most common performance indices used to indirectly optimize the accuracy of a robot are the dexterity index (Gosselin, 1992), the condition numbers, and the global conditioning index (Gosselin and Angeles, 1991).

Another way to estimate the accuracy of a robot is to use the first order approximation of the direct kinematic model that maps the input error vector $\delta \mathbf{q}$ to the output error vector $\delta \mathbf{p}$ through the linear relation $\delta \mathbf{p}=\mathbf{J} \delta \mathbf{q}$, where $\mathbf{J}$ is the Jacobian matrix of the robot. However, such an approach will only give a rough estimate of the maximal errors occurring in the workspace. This estimate is relevant for infinitesimal errors and far from singularities, but on real robots, errors are not always small.

Furthermore, all of the above tools essentially take into account only errors in the inputs. Yet, errors are due to various factors such as manufacturing tolerances, backlash, compliance, sensor errors and control er- 
rors. Besides, once a prototype is built, it is important to be able to simply measure and quantify these errors, which is obviously not done using the Jacobian matrix of the robot.

Clearly, from the industrial point of view, the best accuracy measure would be the maximum translational and maximum rotational errors over a given portion of the workspace (Briot and Bonev, 2008; Merlet, 2006a) or at a given nominal configuration. However, while representing the accuracy for planar robots is simple to realize (Briot and Bonev, 2008), this problem is much more complicated when the number of orientational DOFs increases.

Several papers deal with the accuracy of robots with several orientational DOFs (Jelenkovic and Budin, 2002; Kim and Choi, 2000; Merlet and Daney, 2007; Ropponen and Arai, 1995; Wang and Ehmann, 2002; Zhao et al., 2002). In all of these works, the authors analyse the rotational accuracy of robots by considering the errors on three Euler angles. However, such an analysis is wrong because these errors depend on the choice of reference frame in which the nominal pose and the actual one are expressed. Yet, obviously, a rotational error should depend only on the "distance" between the nominal orientation and the actual one.

For the design of mechanisms, defining metrics for measuring the distance between one pose (position and orientation) and another is of utmost importance and is still an area of ongoing research (Larochelle et al., 2007). For representing the accuracy of a robot in industry, however, it is meaningless to combine translational and rotational errors in a single measure. Fortunately, for the specific case of body orientations, there exists a family of bi-invariant metrics (Park, 1995; Gupta, 1997). Certainly, the most intuitive one is the rotation angle about a unique axis that brings one reference frame to coincide with another.

Single-parameter invariant measures of rotational error are relevant only for asymmetric robot end-effectors. However, robot end-effectors usually have axial symmetry (in most industrial robots, which are sold without an end-effector, the flange on which an end-effector is mounted is axisymmetric). That is why, in this paper, we propose a pair of rotational error measures that is better suited for such end-effectors. These measures are not invariant because their raison d'être is to depend on an end-effector reference frame that takes into account the axial symmetry of the end-effector. However, our measures depend only on the orientation of the actual reference frame with respect to the nominal one.

The remainder of this paper is organized as follows. Section 2 deals with the definition of the proposed measure for orientational accuracy. In Section 3, the new error measure is illustrated on a well-known spherical robot called the Agile Eye. Conclusions are given in the last section. 


\section{Representation of Rotational Errors}

To describe the orientation of a body, three consecutive rotations about some of the three axes of the base reference frame are generally used. The angles of these rotations are referred to as Euler angles.

As previously mentioned, the offsets between the corresponding Euler angles associated with two reference frames varies significantly with the choice of base reference frame that is used to define these Euler angels. Therefore, such an offset cannot be used for measuring rotational errors. As a matter of fact, the same is true when considering the individual $x, y$ and $z$ translational errors, though these errors are bounded by the distance between the origins of the first two reference frames. This distance is, in fact, used in industry to represent translational errors, which is the only accuracy measure currently used (ISO 9283 standard).

Thus, it would be great if a similar invariant metric for the "distance" between two orientations existed. Such metrics exist and the best known one is the rotation angle about a unique axis that brings one reference frame to coincide with another. This angle is not only physically meaningful but also quite easy to compute from the trace of the rotation matrix that represents the orientation of one reference frame with respect to the other.

The only problem with such single-parameter invariant metrics is that robot end-effectors are typically axisymmetric. In some cases, when axisymmetric tools are used, the rotation about the tool axis is even irrelevant. Therefore, if industry ever becomes interested in rotational errors, it will more likely look for a two-parameter metric-one measure that reflects the deviation of the axis of symmetry and, for example, another that reflects the rotation about this axis of symmetry.

Consider two different orientations of an axisymmetric robot endeffector: a nominal one denoted by the index 0 , and another one denoted by the index 1 . Let $\mathbf{d}_{0}$ and $\mathbf{d}_{1}$ be two unit vectors parallel to the axis of symmetry of the robot end-effector in each of the two orientations (Fig. 1). The first meaningful parameter characterizing the change of orientations is clearly the angle $\alpha$ between vectors $\mathbf{d}_{0}$ and $\mathbf{d}_{1}$. Its expression is trivial:

$$
\alpha=\cos ^{-1}\left(\mathbf{d}_{0}^{T} \mathbf{d}_{1}\right)
$$

Now, consider that we render the axes of symmetry of both endeffectors of Fig. 1 coincident by rotating end-effector 1 about an axis $\mathbf{v} 0$ normal to $\mathbf{d}_{0}$ and $\mathbf{d}_{1}$. The smallest angle to which end-effector 1 needs to be rotated about the $\mathbf{d}_{0}$-axis in order to coincide with end-effector 0 will be denoted by $\beta$ and will be our second error measure. 


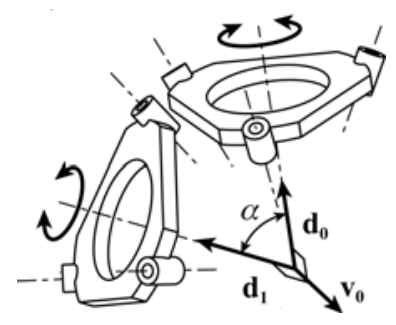

Figure 1. Measuring the rotation errors of a robot end-effector.

Of course, while the choice for the first measure is pretty obvious and unquestionable, there might be other alternatives for the second one. However, we believe that our choice is the most logical and intuitive one.

It can be shown that angles $\alpha$ and $\beta$ are actually the tilt and torsion angles (to a sign difference) of the Tilt-and-Torsion (T\&T) three-angle orientation parameterization introduced in (Bonev, 2002). Therefore, angle $\beta$ can be found using the following equation:

$$
\beta=\left\{\begin{array}{l}
\left|\operatorname{atan} 2\left(r_{3,2} / \sin \alpha,-r_{3,1} / \sin \alpha\right)+\operatorname{atan} 2\left(r_{2,3} / \sin \alpha, r_{1,3} / \sin \alpha\right)\right|, \text { if } \alpha \neq 0 \\
\left|\operatorname{atan} 2\left(r_{2,1}, r_{1,1}\right)\right|, \text { if } \alpha=0
\end{array}\right.
$$

where $r_{i, j}(i, j=1,2,3)$ are the elements of the rotation matrix that represents the orientation of the reference frame associated with the real pose with respect to the reference frame associated with the nominal pose. Note, that $\beta$ does not depend on the choice of the $x$ and $y$ axes.

Obviously, $0 \leq \alpha<90^{\circ}$ in the context of accuracy analysis. In this range, angles $\alpha$ and $\beta$ are the direct equivalent of the translational and rotational errors, respectively, associated with planar 3-DOF motion.

\section{Example: the Agile Eye}

In this section, for simplicity, we address the problem of computing the rotational accuracy of a parallel robot in the presence of active-joint errors only. Specifically, we will analyse the rotational accuracy of the well known spherical parallel robot called the Agile Eye (Fig. 2a). Its kinematics, singularities (Fig. 2b), workspace, and working and assembly modes have been studied in detail in several papers (Bonev and Gosselin, 2005; Bonev and Gosselin, 2006; Bonev et al., 2006). Therefore, we will recall only one important fact before focusing on the accuracy analysis.

The Agile Eye is a very particular parallel robot with its Type 1 (serial) and Type 2 (parallel) singularities coinciding and degenerating to six curves as shown in Fig. 2b using a T\&T angles coordinate system. Each curve corresponds to finite self motions of both the platform and the legs. 


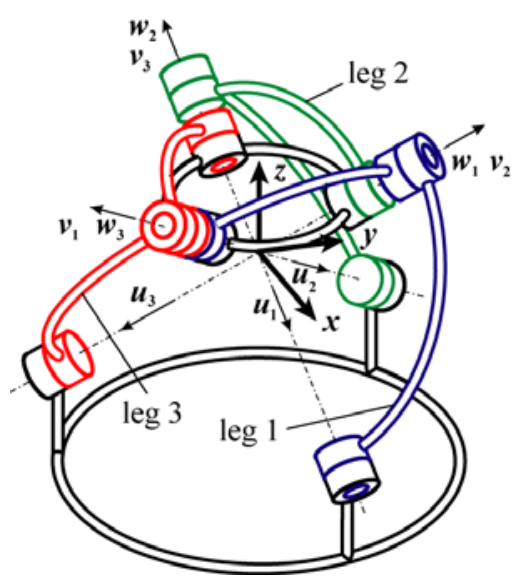

(a)

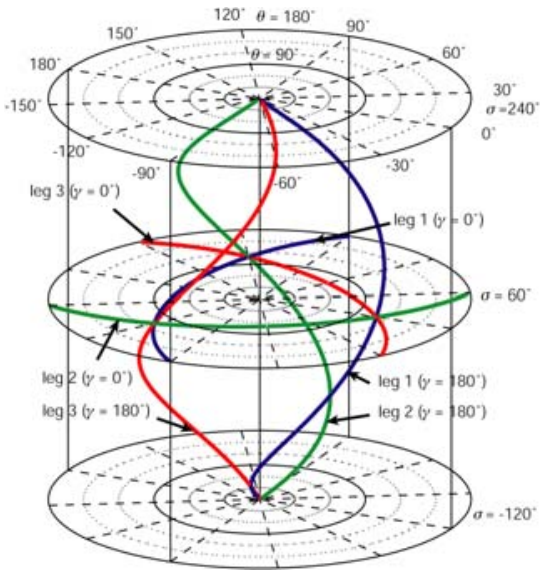

(b)

Figure 2. (a) A CAD model of the Agile Eye at its reference configuration (zero active-joint variables and $\phi=0^{\circ}, \theta=0^{\circ}$ and $\sigma=60^{\circ}$ T\&T angles) and (b) its singularity loci.

\subsection{Accuracy Analysis}

In order to compute the rotational errors of the Agile Eye, we use T\&T angles $(\phi, \theta, \sigma)$ and the following discretization method. For a fixed torsion angle $\sigma$, we discretize the orientation space $(\phi, \theta)$. At a given nominal orientation $\left(\phi_{0}, \theta_{0}, \sigma_{0}\right)$ of the mobile platform, we compute the active-joint variables $\left(q_{10}, q_{20}, q_{30}\right)$ for one given working mode using the inverse kinematics. Clearly, we should stick to the same working mode throughout the whole workspace. Then, assuming that active-joint variable $q_{i}$ can vary from its nominal value $q_{i 0}$ by an error $\varepsilon$ (in our study, $\varepsilon= \pm 0.01^{\circ}$ ), we discretize the active-joint interval from $q_{i 0}-\varepsilon$ to $q_{i 0}+\varepsilon$ and for each set of active-joint variables solve the direct kinematics. For the Agile Eye, the direct kinematics can be found analytically. As presented in (Bonev et al., 2006), there are four non-trivial solutions which define the assembly modes of the robot. Therefore, the problem is to know which of the obtained solutions is the desired assembly mode corresponding to the nominal configuration. Fortunately, this problem has already been solved in (Bonev et al., 2006).

Of course, the direct kinematics of parallel robots can rarely be solved analytically. Therefore, in such cases, a numerical method should be used, such as the Newton-Raphson algorithm. This method is much more time-consuming, but, when sufficiently far from singularities, it converges to the appropriate assembly mode of the robot. 
Thus, for a given nominal configuration, it is possible to compute all possible rotational errors $\alpha$ and $\beta$, and to retain their maxima. These maximal errors are presented in Fig. 3 using T\&T polar plots for several constant torsions. We can think of these polar plots as the equivalent of constant-orientation polar plots for planar robots.

Analysing Fig. 3, it appears that our rotational errors grow considerably near singularities (represented by crosses and dotted lines in Fig. 3), which is perfectly normal. An unexpected result is, however, the fact that, when computing the rotational errors, most of the time, the maximal output errors occur when the active-joint variables are inside their error intervals, whereas it has been demonstrated in (Briot and Bonev, 2008) that for planar parallel robots, the maximal output errors can only occur when at least two of the active-joint variables are at the extremes of their error intervals. This observation suggests that it should be impossible to find a manageable analytic expression for the output (rotational) errors of even a simple spherical parallel robot such as the Agile Eye. A simple discretization method such as ours will be sufficient but more time-consuming and less accurate. However, more efficient approaches could be used, such as interval analysis (Merlet 2006a).

It should also be noted that the curves are periodic, but not symmetric, which is due to the fact that the output errors depend on the robot's working mode.

\section{Conclusions}

In this paper, we addressed the problem of representing rotational errors of spatial robots. A review of previous works has shown that, most frequently, authors analyse the errors on each Euler angle defining the orientation of a robot end-effector. However, this is generally wrong since an accuracy measure should depend only on the "distance" between the nominal pose and the actual one, and not on the choice of reference frame in which these are expressed. In contrast, several bi-invariant metrics for rotational error exist. However, they are single-parameter and, by definition, disregard the shape of the robot end-effector. Yet, most robot endeffectors are axisymmetric. Therefore, the choice of end-effector reference frame is not arbitrary and users are likely to be interested in more than one rotational error measure. To fill this gap, we propose a pair of measures of rotational errors that is better suited for such robot end-effectors.

In order to present the efficiency of our approach, we applied it to compute the output errors of a well-known spherical parallel robot called the Agile Eye. It was shown that its accuracy depends on the working mode and is poor near singularities. 


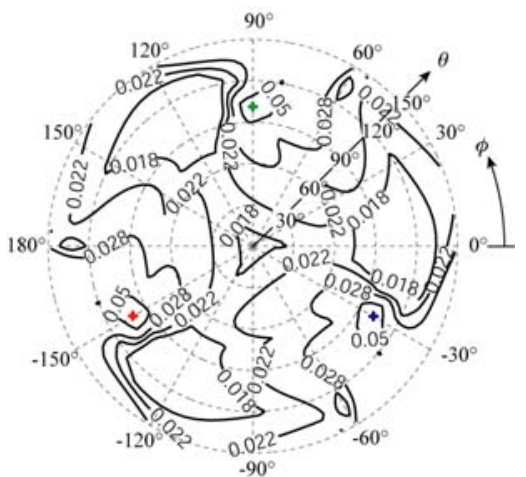

(a) Rotational error $\alpha, \sigma=0^{\circ}$

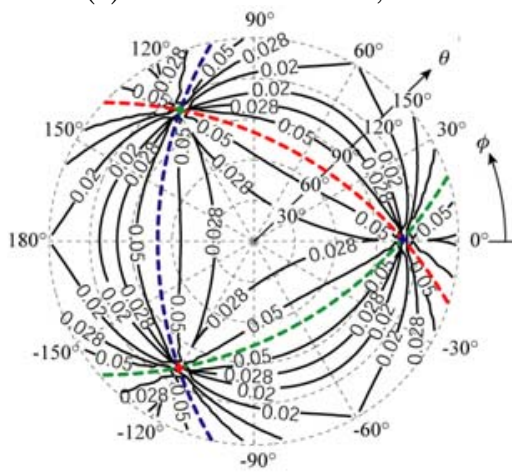

(c) Rotational error $\alpha, \sigma=60^{\circ}$

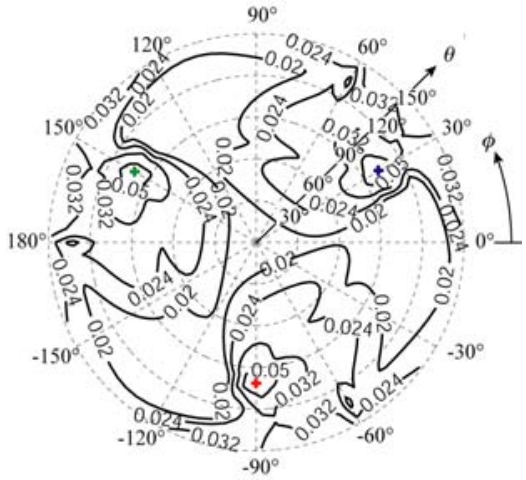

(e) Rotational error $\alpha, \sigma=120^{\circ}$

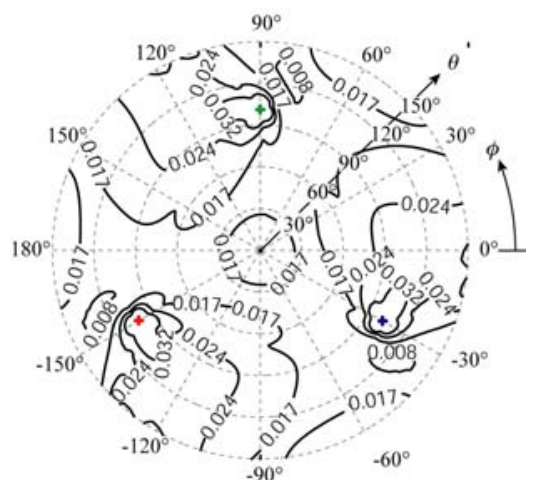

(b) Rotational error $\beta, \sigma=0^{\circ}$

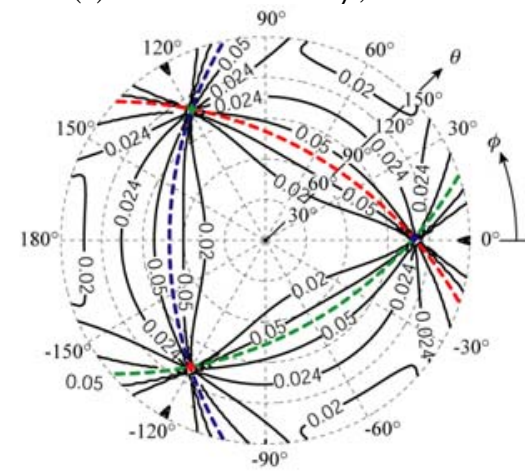

(d) Rotational error $\beta, \sigma=60^{\circ}$

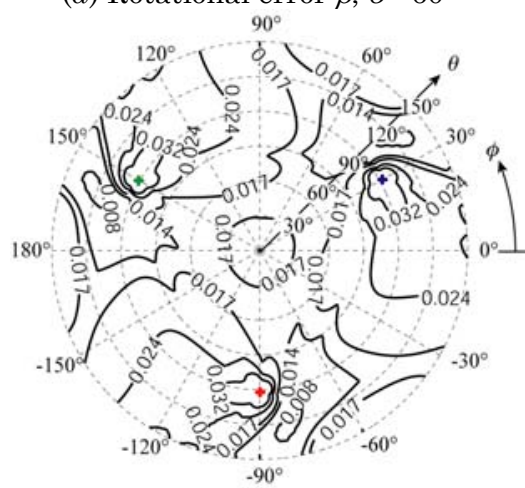

(f) Rotational error $\beta, \sigma=120^{\circ}$

Figure 3. Rotational errors of the Agile Eye for $\sigma \in\left[0^{\circ}, 120^{\circ}\right]$.

\section{Acknowledgements}

We would like to thank the anonymous reviewers for their valuable remarks that helped us improve considerably this manuscript. 


\section{References}

Bonev, I.A. (2002), Geometric Analysis of Parallel Mechanisms, doctoral thesis, Laval University, Quebec, QC, Canada

Bonev, I.A., and Gosselin, C.M. (2005), Singularity loci of spherical parallel mechanisms, Proceedings of the 2005 IEEE Int. Conf. on Robotics and Automation, Barcelona, Spain.

Bonev, I.A., Chablat, D., and Wenger, P. (2006), Working and assembly modes of the Agile Eye, Proceedings of the 2006 IEEE Int. Conf. on Robotics and Automation, Orlando, Florida, USA, pp. 2317-2322.

Bonev, I.A., and Gosselin, C.M. (2006), Analytical determination of the workspace of symmetrical parallel mechanisms, IEEE Transactions on Robotics, vol. 2, no. 5, pp. 1011-1017.

Briot, S., and Bonev, I.A. (2008), Accuracy analysis of 3-DOF planar parallel robots, Mechanism and Machine Theory, vol. 32, no. 4.

Gosselin, C.M., and Angeles, J. (1991), A global performance index for the kinematic optimization of robotic manipulators, Journal of Mechanical Design, vol. 113 , no. 3, pp. 220-226.

Gosselin, C.M. (1992), The optimum design of robotic manipulators using dexterity indices, Robotics and Autonomous Systems, vol. 9, no. 4, pp. 213-226.

Gupta, K.C., Measures of positional error for a rigid body, Journal of Mechanical Design, vol. 119, pp. 346-348.

Jelenkovic, L., and Budin, L. (2002), Error analysis of a Stewart platform based manipulators, Proceedings of the Int. Conf. on Intelligent Engineering Systems (INES), Opatija, Croatia.

Kim, H.-S., and Choi, Y.-J. (2000), The kinematic error bound of the Stewart platform. Journal of Robotic Systems, vol. 17, no. 1, pp. 63-73.

Larochelle, P.M., Murray, A.P., and Angeles, J., A distance metric for finite sets of rigid-body displacements via the polar decomposition, Journal of Mechanical Design, Vo. 129, pp. 883-886.

Merlet, J.-P., and Daney, D. (2005), Dimensional synthesis of parallel robots with a guaranteed given accuracy over a specific workspace, Proceedings of the 2005 IEEE Int. Conf. on Robotics and Automation, Barcelona, Spain, pp. 942-947.

Merlet, J.-P. (2006a), Computing the worst case accuracy of a PKM over a workspace or a trajectory, Proceedings of the $5^{\text {th }}$ Chemnitz Parallel Kinematics Seminar, Chemnitz, Germany, pp. 83-96.

Merlet, J.-P. (2006b), Jacobian, manipulability, condition number, and accuracy of parallel robots, Journal of Mechanical Design, vol. 128, pp. 199-205.

Park, F.C., (1995), Distance metrics on the rigid-body motions with applications to mechanical design, Journal of Mechanical Design, vol. 117, pp.48 -54.

Ropponen, T., and Arai, T. (1995), Accuracy analysis of a modified Stewart platform manipulator, Proceedings of the 1995 IEEE Int. Conf. on Robotics and Automation, Nagoya, Japan, pp. 521-525.

Wang, S.-H., and Ehmann, K.F. (2002), Error model and accuracy analysis of a six-DOF Stewart platform. Journal of Mechanical Design, vol. 124, pp. 286-295.

Zhao, J.-W., Fan, K.-C., Chang, T.-H., and Li, Z. (2002), Error analysis of a serialparallel type machine tool, The International Journal of Advanced Manufacturing Technology, vol. 19, no. 3, pp. 174-179. 